\title{
A model of Stokesian peristalsis and vesicle transport in a three-dimensional closed cavity
}

\author{
Vivian Aranda ${ }^{\mathrm{a}}$, Ricardo Cortez $^{\mathrm{b}}$, Lisa Fauci ${ }^{\mathrm{b}}$ \\ ${ }^{a}$ Department of Civil Engineering, Universidad Técnica Federico Santa María, Av. \\ Vicuña Mackenna 3939, San Joaquin, Santiago, Chile \\ ${ }^{b}$ Department of Mathematics, Tulane University, 6823 St. Charles Avenue, New \\ Orleans, LA 70118, USA
}

\section{Abstract}

The complexity of the mechanics involved in the mammalian reproductive process is evident. Neither an ovum nor an embryo is self-propelled, but move through the oviduct or uterus due to the peristaltic action of the tube walls, imposed pressure gradients, and perhaps ciliary motion. Here we use the method of regularized Stokeslets to model the transport of an ovum or embryo within a peristaltic tube. We represent the ovum or embryo as a spherical vesicle of finite volume - not a massless point particle. The outer membrane of the neutrally-buoyant vesicle is discretized by nodes that are joined by a network of springs. The elastic moduli of these springs are chosen large enough so that a spherical shape is maintained. For simplicity, here we choose an axisymmetric tube where the geometry of the two-dimensional cross-section along the tube axis reflects that of the sagittal cross-section of the uterine cavity. Although the tube motion is axisymmetric, the presence of the vesicle within the tube requires a fully three-dimensional model. As

Email addresses: vivian.aranda@usm.cl (Vivian Aranda), rcortez@tulane.edu (Ricardo Cortez), fauci@tulane.edu (Lisa Fauci) 
was found in $[20,19]$ for a $2 \mathrm{D}$ closed channel, we find that the flow dynamics in a $3 \mathrm{D}$ peristaltic tube are strongly influenced by the closed end and the manner in which the peristaltic wave damps out towards the closure. In addition, we demonstrate that the trajectory of a vesicle of finite volume can greatly differ from the trajectory of a massless fluid particle initially placed at the vesicle's centroid.

Keywords: Ovum transport, peristalsis, Stokes equations, regularized

Stokeslets

\section{Introduction}

Rhythmic muscular contractions that transport fluid occur in most physiological systems. Peristalsis is an example of such a rhythm whereby a wave of contraction is passed along a tube containing fluid. Peristaltic waves convey urine from the kidney to the bladder [13], and move an ingested food bolus along the esophagus towards the stomach [2]. In the reproductive system, oviductal peristalsis contributes to ovum transport to the site of fertilization, and uterine peristalsis enhances sperm transport through the uterus towards the oviduct [12]. The successful implantation of an embryo at the fundal end of the uterus is a crucial stage of mammalian reproduction. Whether the embryo is introduced into the uterus after natural conception or by a catheter after in-vitro fertilization, the intrauterine fluid flow induced by peristalsis could play a central role in this success [4]. In an effort to quantify the effects of uterine peristalsis on embryo transport, researchers have developed a series of fluid dynamic models that analyze the flow induced by peristalsis in a 
two-dimensional channel $[9,10,20]$. Resulting flow rates and trajectories of fluid tracers depend upon the geometry of the channel, the asymmetry of the peristaltic wave and the boundary conditions imposed on the channel ends. In addition, computational models that include a fluid-filled catheter inserted into either an open channel [18] or a closed-end channel [19] show that particle transport depends upon the delivery speed of the catheter load and the timing of catheter discharge with respect to the phase of the peristaltic wave.

Motivated by the model presented in [20] that demonstrated that a closed fundal end dramatically reduced the transport of fluid tracer particles, here we present a model of peristalsis in a three-dimensional closed-end tube. We assume that the length and velocity scales are such that the flow is governed by the Stokes equations of zero Reynolds number flow. As in our previous study of peristaltic pumping [1], the flow is computed using the method of regularized Stokeslets [7]. Moreover, we also include the coupling of an elastic vesicle to the surrounding viscous fluid to model the transport of an embryo of non-zero volume.

In the following sections, we will describe the mathematical formulation of the coupled tube-vesicle-fluid system, and outline the regularized Stokeslet algorithm used to evolve the flow and the vesicle trajectories. We will present numerical results that shed light on the effect of the tube's closed-end on tracer and vesicle transport, and the effect of vesicle radius on overall transport. In addition, we show that a vesicle placed off the centerline of the axisymmetric tube undergoes appreciable rotation as it moves towards the fundal end of the tube. 


\section{Model}

\subsection{Tube dynamics}

We consider a finite, axisymmetric tube whose longitudinal axis is the segment $0 \leq x \leq L$. A cylinder of radius $r_{0}$ is perturbed by a time-dependent peristaltic wave resulting in circular cross sections at a fixed value of $x$ of radius:

$$
r(x, t)=r_{o}+a \sin \left[2 \pi\left(\frac{x}{\lambda}-\frac{t}{T}\right)\right] \tanh \left[\alpha\left(\frac{L}{\lambda}-\frac{x}{\lambda}\right)\right]
$$

where $r_{o}$ is the average tube radius, $a$ is the maximal peristaltic amplitude, $\lambda$ is the wavelength, and $c=\lambda / T$ is the wave velocity. Note that the amplitude of the peristaltic wave is zero at the tube end $x=L$, and the tapering to this zero-amplitude is controlled by the parameter $\alpha$. We choose the tube parameters and wave kinematics in Table 1 based upon those of a sagittal cross-section of a uterine cavity [9]. Figure 1 shows the geometry of this tube at a fixed phase of the peristaltic wave.

\subsection{Vesicle geometry}

The embryo will be represented by a spherical, fluid-filled vesicle whose surface supports elastic forces. The surface membrane of this vesicle will be discretized by nodes that are nearly equally spaced. The surface nodes are connected by a network of Hookean springs with prescribed elastic moduli. If these springs are stretched or compressed away from their resting lengths, restoring forces on the vesicle surface develop. Assume that $\mathbf{y}_{k}$ and $\mathbf{y}_{l}$ are 
two nodes on the vesicle surface that are connected by a linkage or spring of rest length $\Delta_{k l}$. The force at $\mathbf{y}_{k}$ due to this spring is:

$$
\mathbf{g}\left(\mathbf{y}_{k}\right)=\tau_{k l}\left(\frac{\left\|\mathbf{y}_{l}-\mathbf{y}_{k}\right\|}{\Delta_{k l}}-1\right) \frac{\left(\mathbf{y}_{l}-\mathbf{y}_{k}\right)}{\left\|\mathbf{y}_{l}-\mathbf{y}_{k}\right\|}
$$

There is an equal and opposite force due to this spring at the node $\mathbf{y}_{l}$. Here $\tau_{k l}$ is the elastic modulus of the linear spring and $\Delta_{k l}$ is its resting length. Note that the force given in Equation (2) is the product of the elastic modulus of the spring and its relative elongation, and points in the direction of the vector connecting the spring's endpoints. The coupling of these forces to the surrounding viscous fluid is described below.

\subsection{Governing equations of the coupled fluid-tube-embryo system}

The surface of the uterine cavity and the surface of the embryo support localized forces that are exerted on the viscous fluid in which they are immersed. The flow is governed by the incompressible Stokes equations:

$$
\begin{aligned}
& \mu \Delta \mathbf{u}=\nabla p-\mathbf{F}(\mathbf{x}, t)-\mathbf{G}(\mathbf{x}, t) \\
& \nabla \cdot \mathbf{u}=0
\end{aligned}
$$

where $t$ is time, $\mu$ is the dynamic viscosity, $\mathbf{u}$ is fluid velocity (a $3 \mathrm{D}$ vector), and $p$ is the pressure. The force densities (force per volume) $\mathbf{F}(\mathbf{x}, t)$ and $\mathbf{G}(\mathbf{x}, t)$ are exerted on the fluid by the uterine cavity walls and the embryo surface, respectively. These force densities are also 3D vectors.

$$
\begin{aligned}
\mathbf{F}(\mathbf{x}, t) & =\int_{\Sigma_{1}} \mathbf{f}(\mathbf{X}(\mathbf{s}, t), t) \delta(\mathbf{x}-\mathbf{X}(\mathbf{s}, t)) d A \\
\mathbf{G}(\mathbf{x}, t) & =\int_{\Sigma_{2}} \mathbf{g}(\mathbf{Y}(\mathbf{r}, t), t) \delta(\mathbf{x}-\mathbf{Y}(\mathbf{r}, t)) d A
\end{aligned}
$$


where $\mathbf{X}$ are points on the uterine cavity surface $\Sigma_{1}, \mathbf{Y}$ are points on the surface of the vesicle $\Sigma_{2}, \mathbf{s}$ and $\mathbf{r}$ are two-dimensional surface coordinates, and $\delta$ is the three-dimensional Dirac delta function. While the force per unit area distributed on the vesicle surface $\mathbf{g}$ is an elastic membrane force density due to surface deformations, the force per unit area $\mathbf{f}$ on the tube surface will be chosen so that the prescribed kinematics (and hence velocities) given in Equation (1) are achieved. The passive, neutrally-buoyant vesicle is required to move at the local fluid velocity, resulting in the boundary condition:

$$
\frac{d \mathbf{Y}(\mathbf{r}, t)}{d t}=\mathbf{u}(\mathbf{Y}(\mathbf{r}, t), t) .
$$

The solution of the coupled Equations (3)-(5) will capture the viscous drag exerted by the fluid on the vesicle.

\section{Methods}

\subsection{Regularized Stokeslets}

When flow is governed by the Stokes equations in three-dimensional freespace, an isolated force density $\mathbf{h}_{k} \delta\left(\mathbf{x}-\mathbf{X}_{k}\right)$ applied at a single point $\mathbf{X}_{k}$ results in the velocity field given by the classical Stokeslet [15]:

$$
\mathbf{u}(\mathbf{x})=\frac{1}{8 \pi \mu}\left(\frac{1}{r_{k}} \mathbf{h}_{k}+\frac{\left(\mathbf{h}_{k} \cdot\left(\mathbf{x}-\mathbf{X}_{k}\right)\right)\left(\mathbf{x}-\mathbf{X}_{k}\right)}{r_{k}^{3}}\right),
$$

where $r_{k}=\left\|\mathbf{x}-\mathbf{X}_{k}\right\|$ is the distance between the point at which the velocity is evaluated and the point where the force is applied. Due to the linearity of the Stokes equations, the resulting velocity field due to a sum of such point forces would be the sum of the resulting Stokeslets. Although the Stokeslet solution is singular at the points $\mathbf{X}_{k}$ at which forces are applied, when these forces are 
distributed on a two-dimensional surface, the singularity is integrable on that surface. The method of regularized Stokeslets ([7]) replaces the singular point force density with a regularized concentrated force density $\mathbf{h}_{\mathbf{k}} \phi_{\epsilon}\left(\left\|\mathbf{x}-\mathbf{X}_{k}\right\|\right)$ that distributes the force in a small fluid volume around the point. For the choice of the blob (or cut-off) function:

$$
\phi_{\epsilon}(r)=\frac{15 \epsilon^{4}}{8 \pi\left(r^{2}+\epsilon^{2}\right)^{7 / 2}},
$$

the resulting velocity field due to $N$ such regularized forces $\mathbf{h}_{k}$ is given by:

$$
\mathbf{u}(\mathbf{x})=\frac{1}{8 \pi \mu} \sum_{k=1}^{N} \frac{r_{k}^{2}+2 \epsilon^{2}}{\left(r_{k}^{2}+\epsilon^{2}\right)^{3 / 2}} \mathbf{h}_{k}+\frac{\left(\mathbf{h}_{k} \cdot\left(\mathbf{x}-\mathbf{X}_{k}\right)\right)\left(\mathbf{x}-\mathbf{X}_{k}\right)}{\left(r_{k}^{2}+\epsilon^{2}\right)^{3 / 2}} .
$$

We note that the velocity field in Equation (7) is an exact solution to the Stokes equations $\mu \Delta \mathbf{u}=\nabla p-\sum \mathbf{h}_{k} \phi_{\epsilon}\left(\left\|\mathbf{x}-\mathbf{X}_{k}\right\|\right)$, is everywhere incompressible, and is defined everywhere. In addition, this expression tends to the classical Stokeslet as the blob parameter $\epsilon$ tends to zero. The evaluation of Equation (7) at each of the $N$ points at which the regularized forces are concentrated results in a linear relationship between these velocities $\mathbf{U}\left(\mathbf{X}_{k}\right)=\mathbf{U}_{k}$ and the forces $\mathbf{h}_{k}$ for $k=1, \ldots N$. While this linear relationship may be directly evaluated to arrive at velocities when forces are specified, the $3 N \times 3 N$ linear system may also be used to solve for the forces $\mathbf{h}_{k}$ that need to be applied at the points $\mathbf{X}_{k}$ to result in specified velocities $\mathbf{U}_{k}$. This feature is central to our numerical method because the kinematics of the tube surface, and hence the velocities of its material points, are specified. On the other hand, the velocities of the material points on the vesicle surface are not specified, but rather the elastic forces due to its surface deformations are. The algorithm that exploits the linearity of the Stokes equations and the reg- 
ularized Stokeslet solutions to evolve the coupled fluid-tube-vesicle system described by Equations (3), (4) and (5) is described below.

\section{The numerical algorithm}

Our goal is to evaluate the transport of the vesicle within the threedimensional tube due to the peristaltic waves being passed along the tube walls. The surface of the tube $\Sigma_{1}$ at time step $n$ is discretized by $N_{t}$ nodes $\mathbf{X}_{k}^{n}$ for $k=1, \ldots N_{t}$. Using the kinematics in Equation (1), the prescribed velocities $\mathbf{U}_{k}^{n}$ may be calculated by differentiation. At time $t=0$ the surface of the neutrally-buoyant vesicle $\Sigma_{2}$ is discretized by the $N_{v}$ nodes $\mathbf{Y}_{j}^{0}$ for $j=1, \ldots N_{v}$. The distance between each pair of nodes is set to be the rest length of a Hookean spring between those nodes $\left(\Delta_{k l}\right.$ in Equation (2)). The time-stepping algorithm proceeds as follows:

Given the vesicle nodes $\mathbf{Y}_{j}^{n}$ for $j=1, \ldots N_{v}$, and the velocities $\mathbf{U}_{k}^{n}$ imposed at the tube surface nodes $\mathbf{X}_{k}^{n}, k=1, \ldots, N_{t}$ :

1. Compute the $N_{v}$ forces $\mathbf{g}_{j}^{n}$ for $j=1, \ldots N_{v}$ at these vesicle points due to the network of Hookean springs using Equation (2).

2. Use Equation (7) to evaluate at the $N_{t}$ tube nodes $\mathbf{X}_{k}^{n}$ the velocities that would be induced by the $N_{v}$ elastic forces $\mathbf{g}_{j}^{n}$ in the absence of any other forces. Call these auxiliary velocities $\overline{\mathbf{U}}_{k}^{n}, k=1, \ldots, N_{t}$.

3. Use the linear system arrived at by evaluating Equation (7) at the $N_{t}$ tube nodes $\mathbf{X}_{k}^{n}$ to solve for the forces $\mathbf{f}_{k}^{n}, k=1, \ldots, N_{t}$ that need to be applied on the tube surface in order to achieve the velocities on the tube $\mathbf{U}_{k}^{n}-\overline{\mathbf{U}}_{k}^{n}$.

4. Given the distribution of forces $\mathbf{f}_{k}^{n}, k=1, \ldots, N_{t}$ and $\mathbf{g}_{k}^{n}, k=1, \ldots, N_{v}$ at the tube surface and the vesicle surface, respectively, use Equation (7) 
to evaluate the velocities at the vesicle nodes $\mathbf{Y}_{j}^{n}, k=1, \ldots, N_{v}$ due to all forces to arrive at $\mathbf{V}_{j}^{n+1}, j=1, \ldots, N_{v}$.

5. Update the position of the vesicle nodes (using Euler's method for simplicity):

$$
\mathbf{Y}_{j}^{n+1}=\mathbf{Y}_{j}^{n}+\Delta t \mathbf{V}_{j}^{n+1}, \quad j=1, \ldots, N_{v}
$$

Here $\Delta t$ is a time-step. In all of the simulations presented here we use 400 time steps per period of oscillation of the tube. For the wave geometries considered here, we have performed convergence tests that demonstrate that this time resolution is more than adequate. In fact, little differences between trajectories of particles were noted when the time-step was increased to one corresponding to 300 time steps per period.

\subsection{Discretization of tube and vesicle surfaces}

Using the tube geometry and wave kinematics in Table 1, we discretize the tube surface by distributing equidistant nodes around circular cross sections with spacing $\Delta s$ such that the distance between cross sections is also approximately $\Delta s$. In the case of a closed-end tube, nodes are placed within the area of the circular cap, distributed so that each node corresponds to an area element of approximately $\Delta s^{2}$, as described in [1]. At these nodes, the

fluid velocity is specified to be zero. In all simulations presented here, we choose $M=101$ cross sections, $\Delta s \approx 0.01 L$, and a blob size of $\epsilon=1.5 \Delta s$. We have performed spatial convergence tests that compare velocity profiles in the tube computed using $M=81,101,126$ cross sections to resolve the tube, in each case choosing the same relationship between the blob parameter and spacing between nodes. The numerical results demonstrate first order 
convergence in tube discretization. Moreover, the velocities computed using the mid-resolution of $M=101$ differ by a maximum of four percent from those computed at the finest resolution of $M=126$.

A Voronoi tesselation of a surface partitions that surface into discrete regions, each with a designated seed point (or generator), such that all points in a region are closer to that region's seed point than any other seed point. Following [8], we use a spherical centroidal Voronoi tessellation to discretize the vesicle surface. This results in a polyhedron that approximates the sphere so that each nodal point corresponds to a surface area no larger than $\Delta s^{2}$. Because we would like the vesicle to remain spherical, we treat the elastic moduli of the Hookean springs connecting surface node points as penalty parameters that are chosen large enough to resist deformation. We measure the vesicle's deformation from a sphere by computing the distance of each surface point to the vesicle's centroid, and then compare these to the prescribed radius. We note that in the simulations presented here, this deformation never exceeded ten percent.

\section{Results}

We present the results of our simulations using nondimensional length and time, where the length scale is chosen to be the wavelength $\lambda$ and the time scale is the temporal period $T$ of the peristaltic wave.

\subsection{Trajectories of tracer (massless) particles}

In [1], we examined the peristaltic pumping of a Stokesian fluid when a phase-shifted asymmetry was imposed upon an open three-dimensional tube. 
In that study, we also validated the numerical method by comparing computed flow rates in an axisymmetric tube with those computed for periodic axisymmetric tubes using long wavelength theory [17]. It was previously shown by [14] that flow rates in open-ended, finite tubes with an integral number of wavelengths are the same as those in the periodic case.

Before addressing vesicle transport, we first examine the trajectories of fluid tracers in axisymmetric tubes of three kinds: (1) open tube, (2) closedend with long taper $(\alpha=1)$ and (3) closed-end with short taper $(\alpha=40)$. In this system where no vesicle is being tracked, we can solve a linear system for the forces that must be applied at the tube surface points (including the circular cap) so that the desired velocities are achieved. The resulting velocity of a tracer particle may then be directly computed by summing the contributions from these forces (Equation (7)). Figure 2 shows the trajectories of five tracer particles initially seeded radially within the circular disk at $x=L / 8 \mathrm{~mm}$. The particle trajectories remain in the plane containing the particles' initial positions and the tube axis due to axial symmetry. The trajectories in Figure 2(a), the open tube, are tracked for 1.5 periods, when those initialized closer to the tube axis have exited the tube. Figures 2(b) and 2(c) show the trajectories of tracers with the same initial positions within the closed-end tubes of long and short taper, respectively. These trajectories are tracked for two periods. While the particles that begin near the tube axis do move towards the closed fundal end, we see that those placed off-center trace out loops but make substantially less progress in the axial direction than their counterparts in the open tube. The manner in which the wave decays at the tube closure also has a significant impact on the trajectories 
(compare Figure 2(b) and Figure 2(c)). The two-dimensional simulations in closed-end channels presented by [20] show qualitatively similar results. Moreover, [20] identified cellular regions within the channel, spaced one wavelength apart, where fluid markers that were initialized within these regions could not escape. We have not identified such impermeable regions in the three-dimensional tube although the manner in which the tube tapers seems to affect the possibility of cellular regions.

\subsection{Trajectories of embryo}

The embryo at the time of implantation in the uterus, called a blastocyst, is not a fluid tracer, but a nearly spherical object of radius approximately $r=0.07 \mathrm{~mm}$ [16]. Elastic vesicles of non-zero volume can span fluid streamlines, and also exert force on the surrounding fluid, influencing the velocities induced by the contracting tube walls. In order to emphasize the effect of a vesicle of non-zero radius, we examine embryos with radii larger than that of a blastocyst, but of the same order of magnitude. We initialize the vesicle so that its centroid is at the cross-section of the tube $x=L / 8$ and is either on the tube axis or off the axis but always in the $x y$-plane. Figure 3 shows the results of twenty four simulations where the vesicles of four different radii $(r=0, r=0.14 \mathrm{~mm}, r=0.21 \mathrm{~mm}, r=0.42 \mathrm{~mm})$ are placed within the three tubes, with centroid either on-axis or off-axis. Note that the vesicle of zero radius is just a fluid tracer. The largest vesicle considered has a radius that is 21 percent of the tube radius. The first row, Figure 3(a,b), shows that in an open tube there is little difference between the $x$-displacement of the vesicles and the point particle whether they start on the axis or off the axis. In both of the closed-end tubes, we see that the vesicles that are initial- 
ized on-axis travel paths that are not much dependent on their radii (Figure $3(\mathrm{c}, \mathrm{e}))$. In fact, the vesicle trajectories are well-approximated by the trajectory of a fluid tracer. These centrally-seeded vesicles advance just about to the tube closure $x=L$ in the short-taper tube, and oscillate near $x=1.7 L$ in the long-taper tube. The dramatic difference between $x$-trajectories of vesicles of non-zero volume and tracer particles occurs for those placed off-axis in the closed-end tubes. The solid curves in Figure $3(\mathrm{~d}, \mathrm{f})$ depicting fluid tracers show that they do not make any progress down the tube, but undergo periodic oscillations, as observed in the simulations of [20]. However, vesicles of non-zero radius are able to move across streamlines, and progress towards the fundal end (dashed curves). In fact, the vesicle with the largest radius moves towards the closed end most quickly. Figure 3(d) also shows how the long taper slows down the progress of the vesicles. Figure 4 shows the three-dimensional trajectories of the fluid tracer and the three vesicles in the short-tapered, closed-end tube. We remark that the tracer particles and the vesicle centroids are initially placed in the $x y$-plane (but off the tube axis) so that they remain in this plane throughout the simulation due to the symmetry of the tube.

We also note that a spherical, non-zero radius embryo initially placed offcenter undergoes significant rotation as it progresses down the tube towards the closed end. Figure 5 shows snapshots of the vesicle of largest radius as it progresses within the long-tapered, closed-end channel. Initially, the centroid of this vesicle was placed just off the axis of the tube, at $(0.25,0.06,0)$, where the wavelength $\lambda$ corresponds to a unit length. The coloring on the panels of the discretized vesicle demonstrates that the rotation changes di- 
rection during the timeframe shown. Because the motion of the tube walls is axisymmetric, the vesicle can only rotate about the normal to the fixed plane containing the tube axis and the centroid of the vesicle at time zero. We show snapshots of the embryo within this plane at time intervals of .125 of a peristaltic period in Figure 6. The rotation of the sphere is characterized by a single angle $\theta(t)$, shown in Figure 7 , where red dots indicate the angle corresponding to the snapshots in Figure 6. Again, we see the change in direction of rotation.

\section{Discussion}

Motivated by embryo transport in the uterine cavity, here we presented a model of peristaltic transport of a vesicle within a three-dimensional tube. We examined such transport in the case of an open axisymmetric tube as well as an axisymmetric tube with a closed end. We find that, in a closedend tube, the transport of a finite-sized vesicle differs from that of a fluid tracer placed at its centroid when the vesicle is introduced off-axis. While fluid tracers do not make progress towards the fundal end of the idealized uterine cavity, the vesicles can cross streamlines and progress towards an implantation site. These differences in trajectories become more pronounced as the radius of the vesicle increases.

Previous computational studies of finite-size particle transport in a twodimensional periodic channel include an immersed boundary model by [11], and a more recent lattice Boltzmann model by [6]. [11] found that an axiallyplaced circular particle was transported in the direction of the peristaltic wave at a velocity much greater than a fluid tracer. This seems to contradict the 
corresponding results presented here for an axially-placed vesicle in an open tube, where we observe very little difference between the transport speeds of tracers and vesicles. However, [6] showed that the increased transport speed observed in [11] was due to the periodic copy of the circular particle at every wavelength of the channel. When periodic channels with only one finite-sized particle per five or ten wavelengths was tracked, the transport speed did not increase with particle diameter ([6]). Again, we see the crucial dependence upon boundary conditions imposed at the tube ends.

This method that couples a vesicle of non-zero volume to a Stokesian fluid has recently been used to investigate the transport of a microscale load by a rotating helical flagellum ([3]). Note that this algorithm does allow the vesicle shape to respond to the evolving fluid flow. Here we treat the elastic moduli of the Hookean springs as penalty parameters chosen large enough to maintain a nearly spherical shape. Alternatively, these may be chosen to reflect the material properties of the vesicle, which could be more deformable.

\section{Conclusion}

In summary, we assert that trajectories of neutrally-buoyant vesicles of small but non-zero radii within a peristaltic tube can be dramatically different from those of massless fluid tracers. This can have implications in the study

of embryo transport in a uterine cavity, and suggests that embryos of finite volume can progress towards the fundus better than fluid particles of zero volume given the same motion of the uterine walls.

We remark that this algorithm does not represent a fully coupled system, since the kinematics of the axisymmetric tube are pre-set and do not 
depend upon the presence of the immersed vesicle. In reality, the surface of the uterine cavity is a complex structure composed of passive elastic tissue and actuated muscles, whose contractions are induced by an interplay of biochemical and electrical signals ([5]). Future models of embryo transport should include a representation of the force-generating mechanisms of the uterine muscles and feedback from the surrounding fluid.

\section{Acknowledgements}

The work of R. Cortez and L. Fauci was supported, in part, by NSF DMS-104626. The work of V. Aranda was supported by UTFSM Research Project $\mathrm{N}^{\circ} 261381$.

\section{References}

[1] Aranda, V., Cortez, R., Fauci, L., 2011. Stokesian peristaltic pumping in a three-dimensional tube with a phase-shifted asymmetry. Phys. Fluids 23, 081901-081910.

[2] Brasseur, J., Nicosia, M., Pal, A., L, M., 2007. Function of longitudinal vs circular muscle fibers in esophageal peristalsis, deduced with mathematical modeling. World J Gastroenterol. 13, 1335-1346.

[3] Buchmann, A., Fauci, L., Leiderman, K., Strawbridge, E., Zhao, L., 
2014. Flow induced by bacterial carpets and transport of microscale loads. IMA Vol. in Math. Appl. in press.

[4] Bulletti, C., de Ziegler, D., 2005. Uterine contractility and embryo implantation. Curr. Opin. Obstetrics and Gynec. 17 (265-276).

[5] Bursztyn, L., Eytan, O., Jaffa, A., Elad, D., 2007. Modeling myometrial smooth muscle contraction. Annals of NY Acad. Sci. 1101, 110-138.

[6] Connington, K., Kang, Q., Viswanathan, H., Abdel-Fattah, A., Chen, S., 2009. Peristaltic particle transport using the lattice boltzmann method. Phys. of Fluids 21, 053301.

[7] Cortez, R., Fauci, L., Medovikov, A., 2005. The method of regularized stokeslets in three dimensions: analysis, validation, and application to helical swimming. Phys. Fluids 17, 031504.

[8] Du, Q., Gunzburger, M., Ju, L., 2003. Constrained centroidal voronoi tessellations for surfaces. SIAM J. Sci. Comput. 24, 1488-1506.

[9] Eytan, O., Elad, D., 1999. Analysis of intra-uterine fluid motion induced by uterine contractions. Bull. Math. Biol. 61, 221.

[10] Eytan, O., Jaffa, A., Elad, D., 2001. Peristaltic flow in a tapered channel: application to embryo transport within the uteriine cavity. Med. Engr. Phys. 23, 473.

[11] Fauci, L., 1992. Peristaltic pumping of solid particles. Comp. \& Fluids $21,583$. 
[12] Fauci, L., Dillon, R., 2006. Biofluidmechanics of reproduction. Annu. Rev. Fluid. Mech. 38, 371-394.

[13] Jimenez-Lozano, J., Sen, M., Dunn, P., 2009. Particle motion in unsteady two-dimensional peristaltic flow with application to the ureter. Phys. Rev. E 79, 041901.

[14] Li, M., Brasseur, J., 1993. Nonsteady peristaltic transport in finitelength tubes. J. Fluid Mech. 248, 129.

[15] Pozrikidis, C., 1992. Boundary integral and singularity methods for linearized viscous flow. Cambridge.

[16] Romao, G., Araujo, M., de Melo, A., Navarro, P., Ferriani, R., Dos Reis, R., 2010. Oocyte diameter as a predictor of fertilization and embryo quality in assisted reproduction cycles. Fertil. Steril. 93 (2), 621.

[17] Shapiro, A., Jaffrin, M., Weinberg, S., 1969. Peristaltic pumping with long wavelengths at low reynolds number. J. Fluid Mech. 37, 799-825.

[18] Yaniv, S., Elad, D., Jaffa, A., Eytan, O., 2003. Biofluid aspects of embryo transfer. Ann. Biomed. Engr. 31, 1255.

[19] Yaniv, S., Jaffa, A., Elad, D., 2012. Modeling embryo transfer in a closed uterine cavity. J. Biomech. Engr. 134, 111003-1.

[20] Yaniv, S., Jaffa, A., Eytan, O., Elad, D., 2009. Simulation of embryo transport in a closed uterine cavity model. Eur. J. Obstet. Gynecol. Reprod. Biol. 144, S50-S60. 


\section{Table}

Table 1: Model parameters

\begin{tabular}{lccl}
\hline Parameter & Symbol & Units & Value \\
\hline Channel length & $L=2 \lambda$ & $m m$ & 50 \\
Wavelength & $\lambda$ & $m m$ & 25.0 \\
Tube radius & $r_{o}$ & $m m$ & 2.0 \\
Amplitude & $a$ & $m m$ & 0.6 \\
Wave speed & $c$ & $\mathrm{~mm} / \mathrm{s}$ & 1.25 \\
Viscosity & $\mu$ & $\mathrm{gm} / \mathrm{mm} \cdot \mathrm{s}$ & 0.001 \\
Frequency & $f$ & $H z$ & 0.05 \\
Period & $T=1 / f$ & $s$ & 20 \\
Short taper parameter & $\alpha_{s}$ & - & 40 \\
Long taper parameter & $\alpha_{l}$ & - & 1 \\
Vesicle radius & $\mathrm{r}$ & $\mathrm{mm}$ & $0.00-0.42$ \\
\hline
\end{tabular}

\section{Figures Legends}

Figure 1: Schematic of the axisymmetric tube with a closed end. The tube surface at $x=L$ is a circular disk.

Figure 2: Trajectories of fluid tracers that were initially seeded radially within the circular disk at $x=L / 8$ in the case of (a) an open tube, (b) a 
closed-end tube with long taper, $\alpha=1$, (c) a closed-end tube with short taper, $\alpha=40$. Note that the peristaltic tube is shown only at its initial phase for reference and that the particles always remain within the peristaltic tube.

Figure 3: Progress of vesicle centroids in the axial direction. (a)Centroids initialized on axis in open tube. (b) Centroids initialized off axis in open tube. (c) Centroids initialized on axis in closed-end, long tapered tube. (d) Centroids initialized off axis in closed-end, long tapered tube. (e) Centroids initialized on axis in closed-end, short tapered tube. (f) Centroids initialized off axis in closed-end, short tapered tube.

Figure 4: Trajectories of vesicles of radii (a) $\mathrm{r}=0.00 \mathrm{~mm}$, (b) $0.14 \mathrm{~mm}$, (c) $0.21 \mathrm{~mm}$, (d) $0.42 \mathrm{~mm}$ in the closed-end tube $\alpha=40$ tracked for 6 periods. The vesicles were initially placed off-axis. These trajectories are the $3 \mathrm{D}$ views of those depicted in Figure 3(f).

Figure 5: Snapshots of vesicle of radius $R$, discretized with $N_{v}=59$ nodes, at four different times within the closed-end, long tapered tube. Zoomedin view of the discretized vesicle surface are shown with panels colored to demonstrate rotation. Note that the direction of rotation does change within the shown time period.

Figure 6: Snapshots of embryo at time intervals of 0.125 in the plane perpindicular to the axis of rotation. This simulation is the same as that in Figure 5. 
Figure 7: Evolution of the angular rotation, showing change in direction. Red dots correspond to times shown in Figure 6.

\section{Figures}

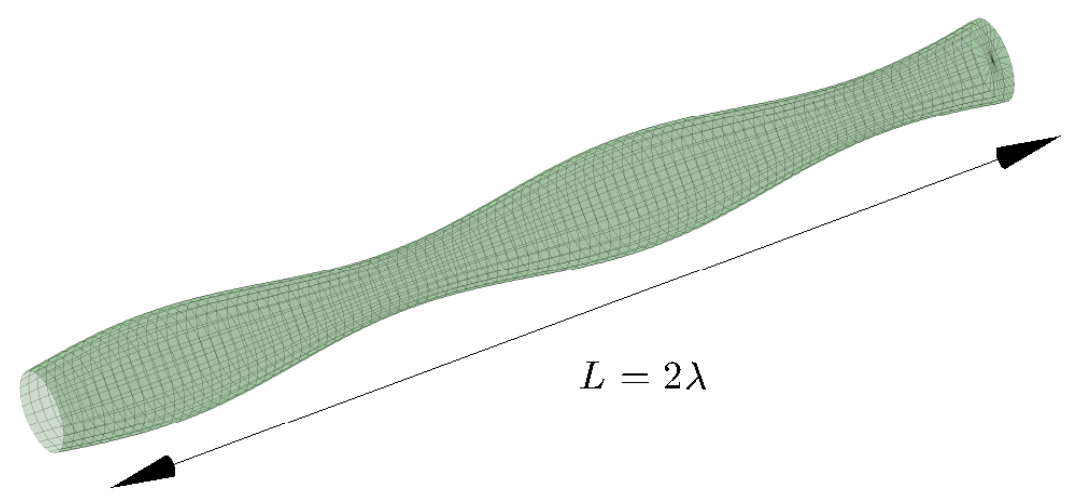

Figure 1: Schematic of the axisymmetric tube with a closed end. The tube surface at $x=L$ is a circular disk. 


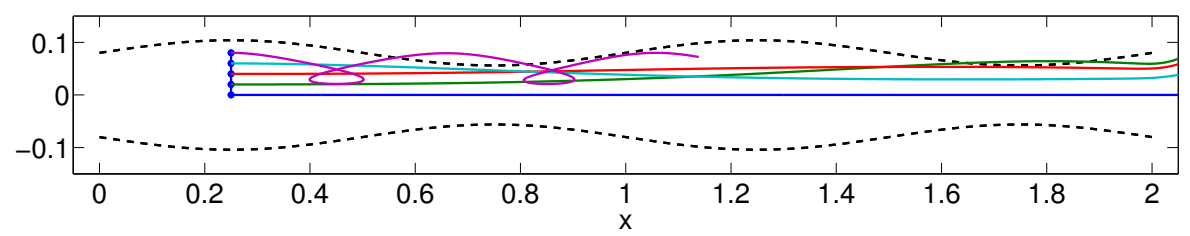

(a) Open tube after 1.5 periods.

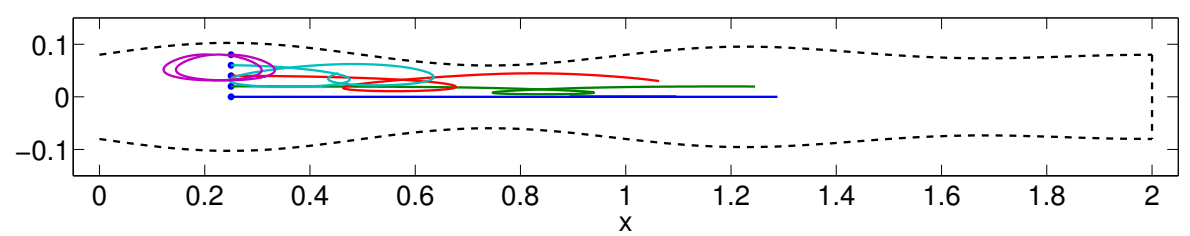

(b) Closed tube after 2 periods, $\alpha=1$

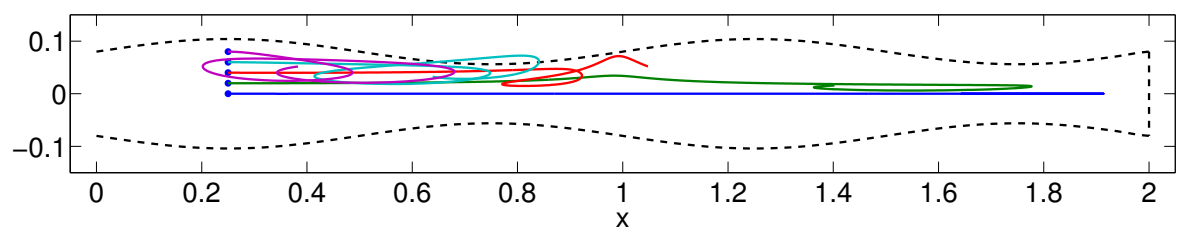

(c) Closed tube after 2 periods, $\alpha=40$

Figure 2: Trajectories of fluid tracers that were initially seeded radially within the circular disk at $x=L / 8$ in the case of (a) an open tube, (b) a closed-end tube with long taper, $\alpha=1$, (c) a closed-end tube with short taper, $\alpha=40$. Note that the peristaltic tube is shown only at its initial phase for reference and that the particles always remain within the peristaltic tube. 


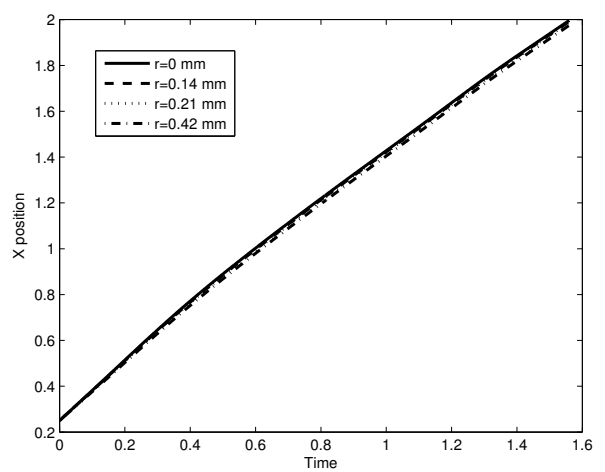

(a) Open tube; on axis.

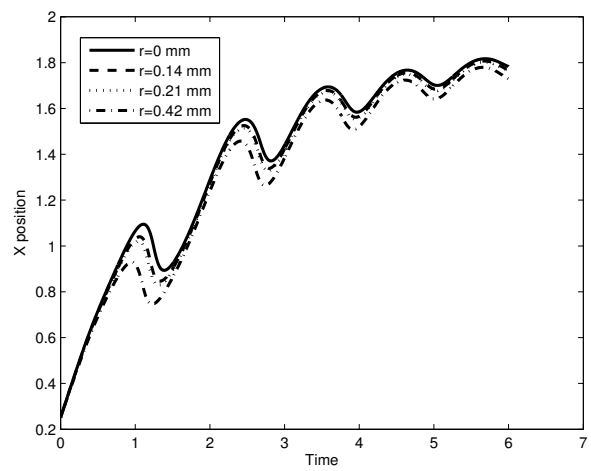

(c) Closed-end $(\alpha=1)$; on axis.

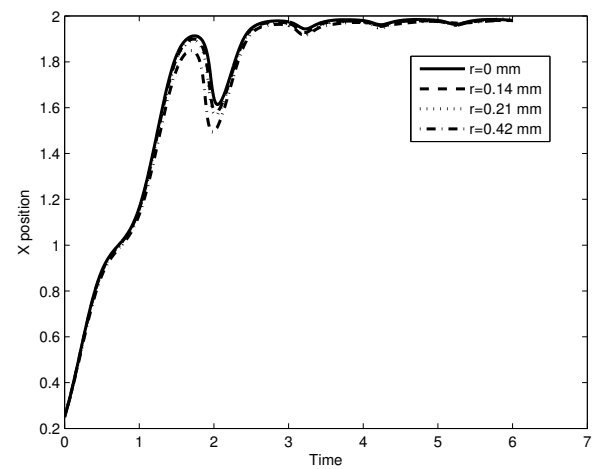

(e) Closed-end $(\alpha=40)$; on axis.

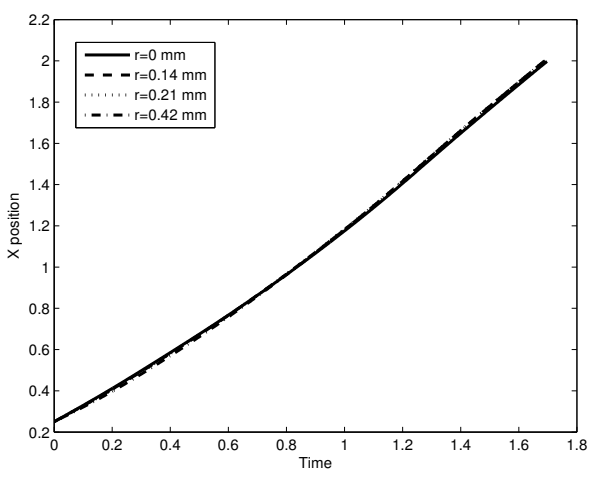

(b) Open tube; off-axis.

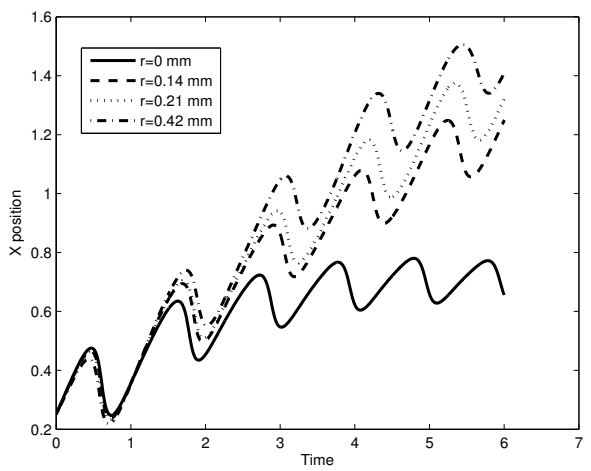

(d) Closed-end $(\alpha=1)$; off-axis.

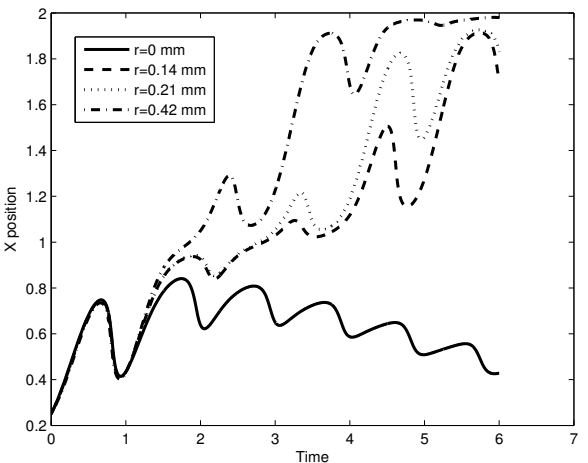

(f) Closed-end ( $\alpha=40)$; off-axis.

Figure 3: Progress of vesicle centroids in the axial direction. (a)Centroids initialized on axis in open tube. (b) Centroids initialized off axis in open tube. (c) Centroids initialized on axis in closed-end, long tapered tube. (23\% Centroids initialized off axis in closed-end, long tapered tube. (e) Centroids initialized on axis in closed-end, short tapered tube. (f) Centroids initialized off axis in closed-end, short tapered tube. 


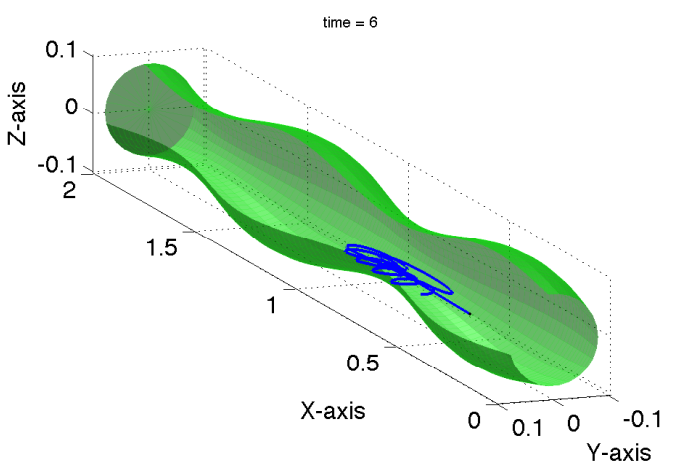

(a) $\mathrm{r}=0.00 \mathrm{~mm}$ (tracer particle)

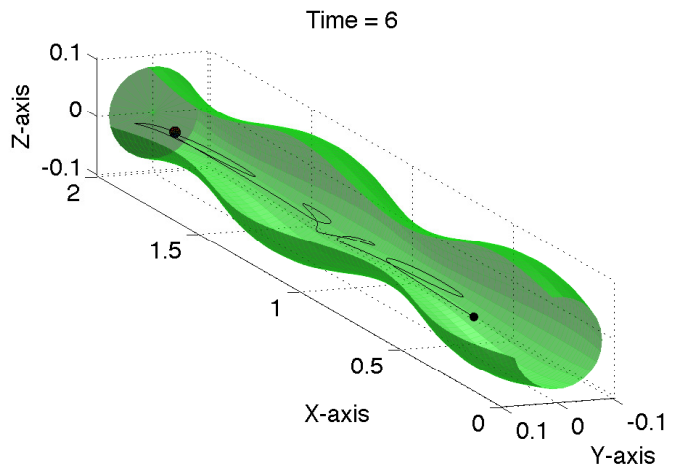

(c) $\mathrm{r}=0.21 \mathrm{~mm}$

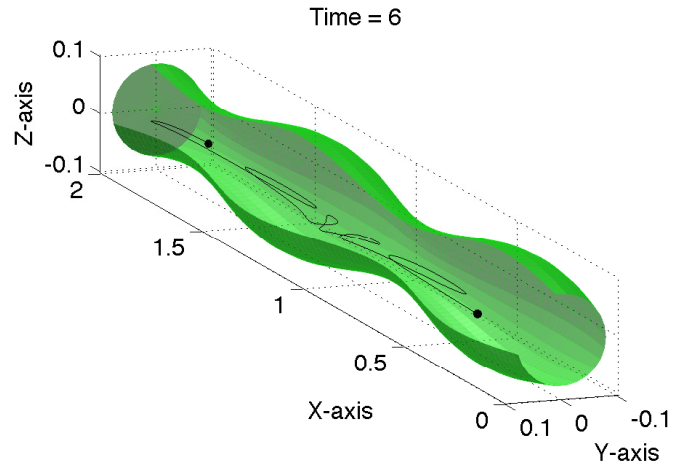

(b) $\mathrm{r}=0.14 \mathrm{~mm}$

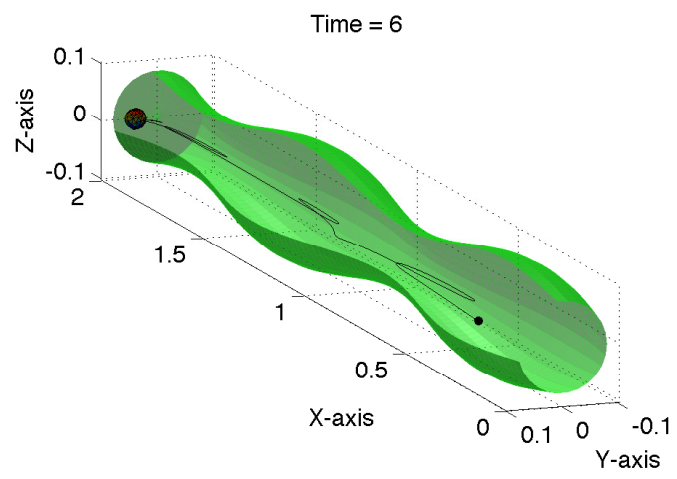

(d) $\mathrm{r}=0.42 \mathrm{~mm}$

Figure 4: Trajectories of vesicles of radii (a) $\mathrm{r}=0.00 \mathrm{~mm}$, (b) $0.14 \mathrm{~mm}$, (c) $0.21 \mathrm{~mm}$, (d) $0.42 \mathrm{~mm}$ in the closed-end tube $\alpha=40$ tracked for 6 periods. The vesicles were initially placed off-axis. These trajectories are the 3D views of those depicted in Figure 3(f). 

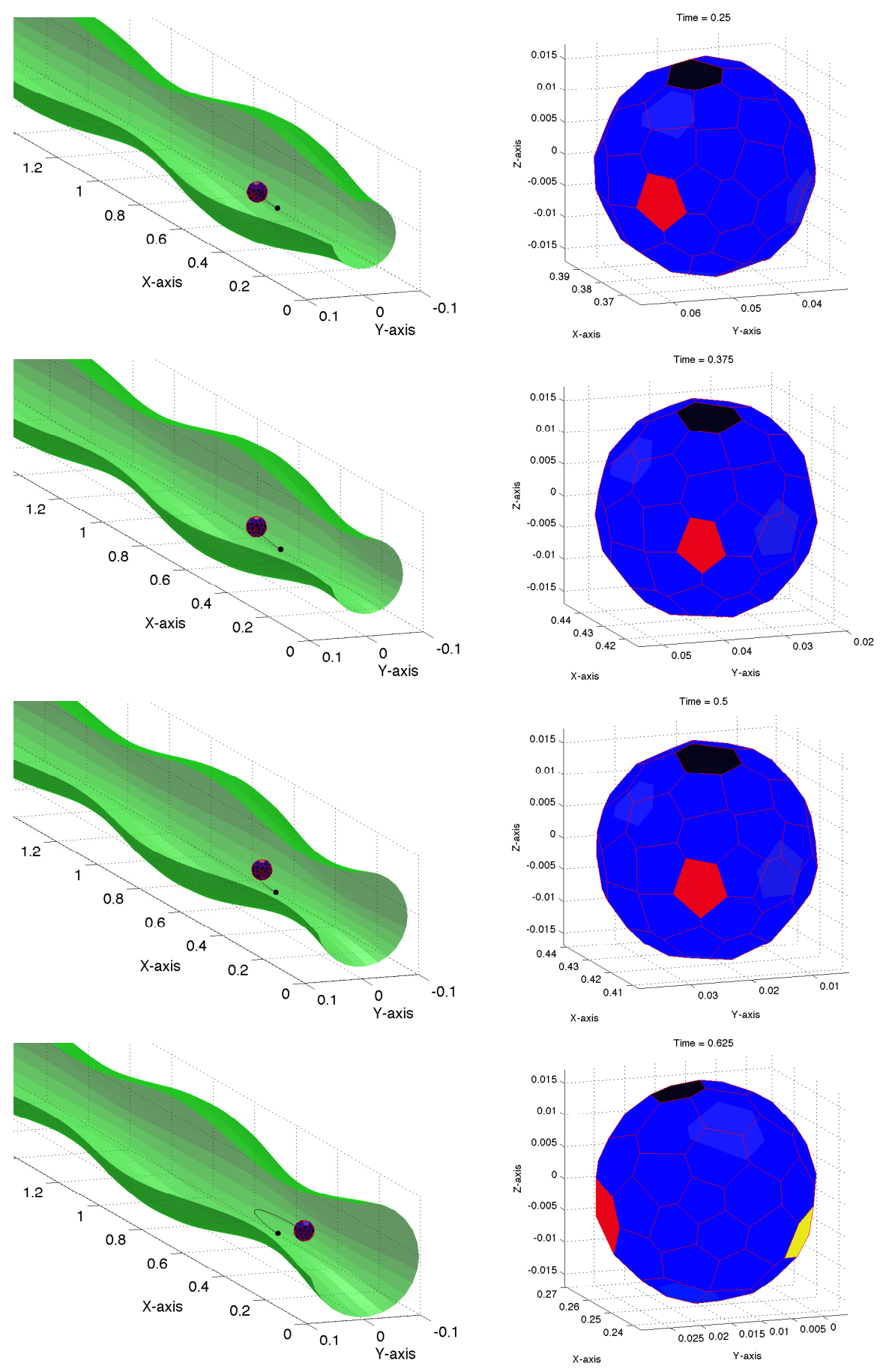

Figure 5: Snapshots of vesicle of radius $R$, discretized with $N_{v}=59$ nodes, at four different times within the closed-end, long tapered tube. Zoomed-in view of the discretized vesicle surface are shown with panels colored to demonstrate rotation. Note that the direction of rotation does change within the shown time period. 
Trajectory of embryo at intervals of time of 0.125

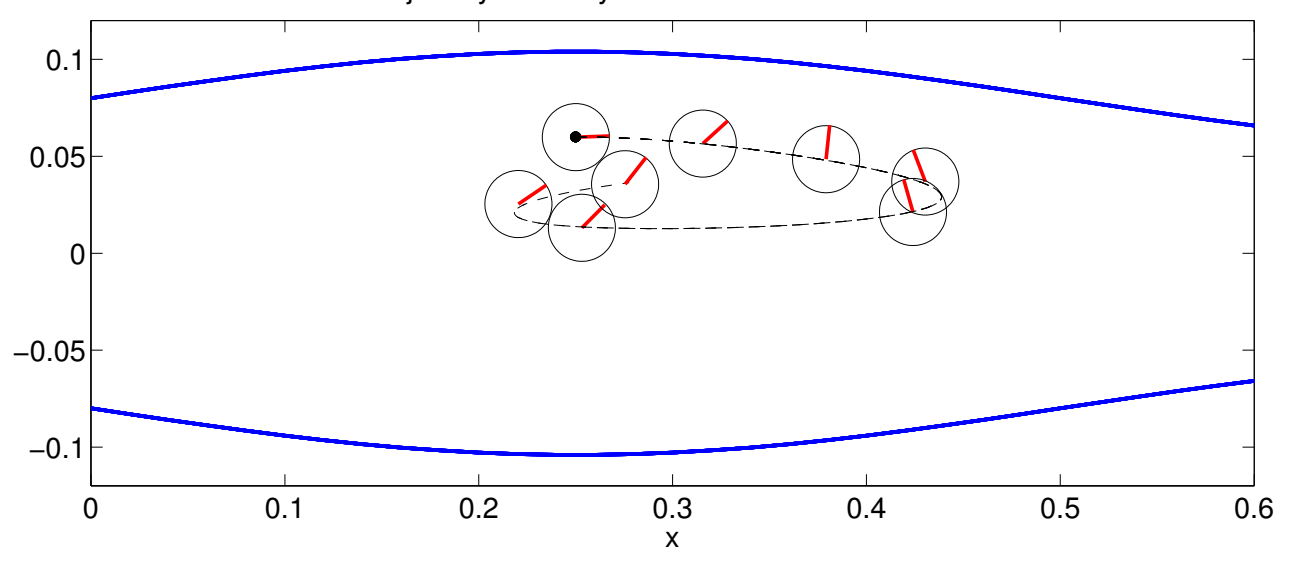

Figure 6: Snapshots of embryo at time intervals of 0.125 in the plane perpindicular to the axis of rotation. This simulation is the same as that in Figure 5. 


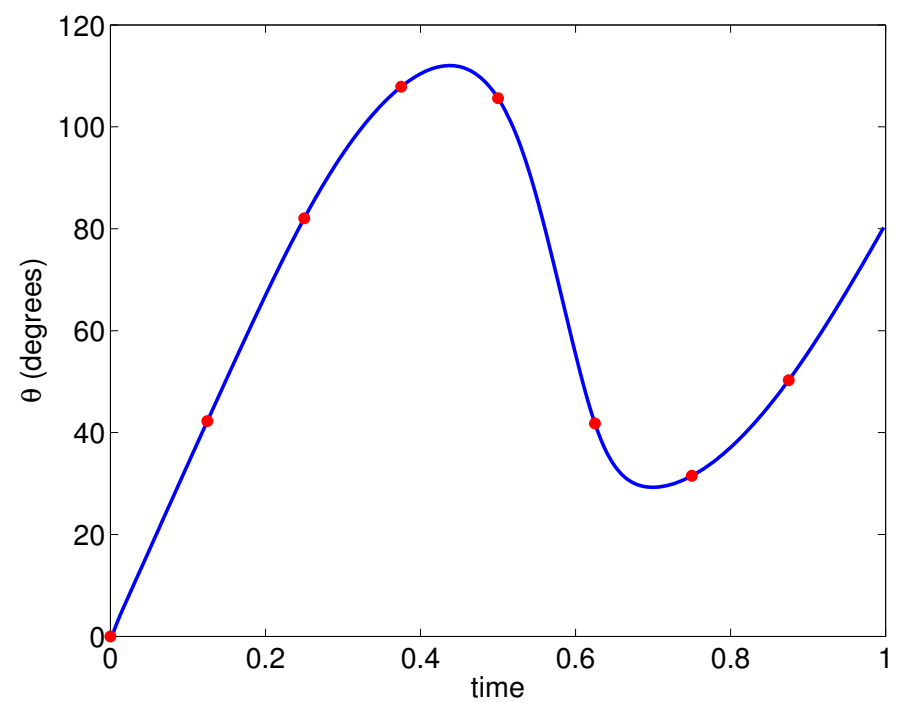

Figure 7: Evolution of the angular rotation, showing change in direction. Red dots correspond to times shown in Figure 6. 